\title{
Nonlinear interaction of meta-atoms through optical coupling
}

A. P. Slobozhanyuk, , P. V. Kapitanova, , D. S. Filonov, , D. A. Powell, , I. V. Shadrivov, , M. Lapine, , P. A. Belov, , R. C. McPhedran, and, and Yu. S. Kivshar

Citation: Appl. Phys. Lett. 104, 014104 (2014); doi: 10.1063/1.4861388

View online: http://dx.doi.org/10.1063/1.4861388

View Table of Contents: http://aip.scitation.org/toc/apl/104/1

Published by the American Institute of Physics

\section{Articles you may be interested in}

Controlling split-ring resonators with light

Applied Physics Letters 99, 251914 (2011); 10.1063/1.3671617

Resonant meta-atoms with nonlinearities on demand

Applied Physics Letters 109, 111904 (2016); 10.1063/1.4962838

Magnetic Purcell factor in wire metamaterials

Applied Physics Letters 104, 161105 (2014); 10.1063/1.4872163

Competing nonlinearities with metamaterials

Applied Physics Letters 101, 231904 (2012); 10.1063/1.4768945

Magnetoinductive waves in one, two, and three dimensions

Journal of Applied Physics 92, 6252 (2002); 10.1063/1.1510945

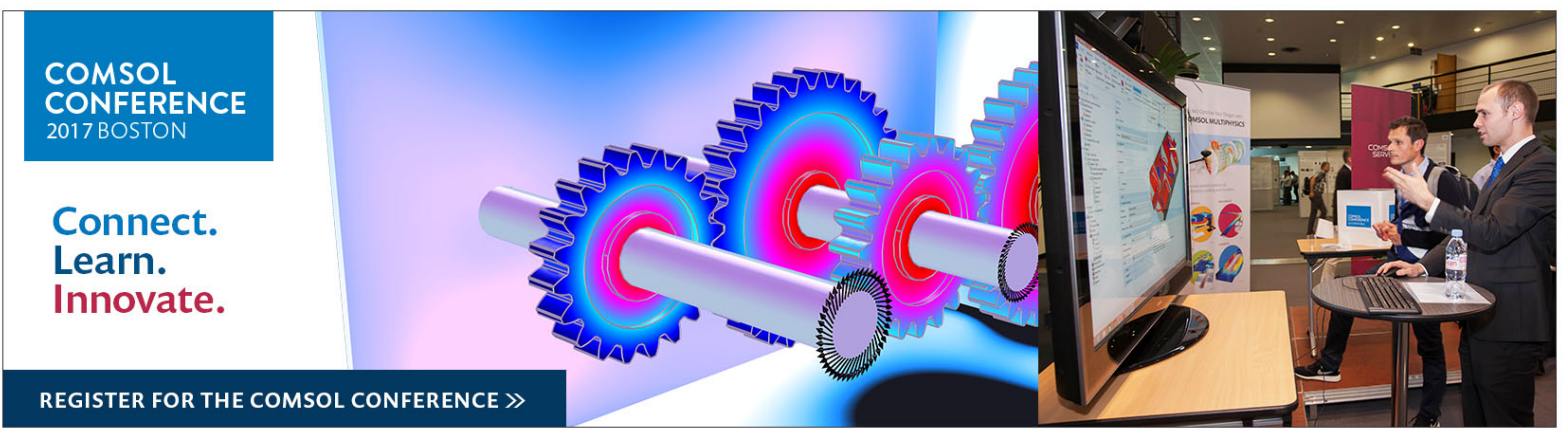




\title{
Nonlinear interaction of meta-atoms through optical coupling
}

\author{
A. P. Slobozhanyuk, ${ }^{1}$ P. V. Kapitanova, ${ }^{1}$ D. S. Filonov, ${ }^{1}$ D. A. Powell, ${ }^{2}$ I. V. Shadrivov, ${ }^{1,2}$ \\ M. Lapine, ${ }^{1,3, a)}$ P. A. Belov, ${ }^{1}$ R. C. McPhedran, ${ }^{3}$ and Yu. S. Kivshar ${ }^{1,2}$ \\ ${ }^{1}$ National Research University of Information Technologies, Mechanics and Optics (ITMO), \\ St. Petersburg 197101, Russia \\ ${ }^{2}$ Nonlinear Physics Centre and Centre for Ultrahigh-bandwidth Devices for Optical Systems (CUDOS), \\ Australian National University, Canberra, ACT 0200, Australia \\ ${ }^{3}$ Centre for Ultrahigh-bandwidth Devices for Optical Systems (CUDOS), School of Physics, University \\ of Sydney, New South Wales 2006, Australia
}

(Received 5 November 2013; accepted 30 November 2013; published online 9 January 2014)

We propose and experimentally demonstrate a multi-frequency nonlinear coupling mechanism between split-ring resonators. We engineer the coupling between two microwave resonators through optical interaction, whilst suppressing the direct electromagnetic coupling. This allows for a power-dependent interaction between the otherwise independent resonators, opening interesting opportunities to address applications in signal processing, filtering, directional coupling, and electromagnetic compatibility. (C) 2014 AIP Publishing LLC. [http://dx.doi.org/10.1063/1.4861388]

Frequency interaction forms the core of nonlinear optics $^{1}$ and has been thriving since the advent of lasers, which gave access to signal powers sufficient to exploit the relatively weak optical nonlinearities. At the same time, microwave engineering has employed complicated circuitry with active devices to realise nonlinear interaction in the radio and microwave domains. ${ }^{2}$ However, the interacting frequencies must usually obey fixed relationships determined by conservation of photon energy, as in three-wave-mixing and harmonic generation, or be linked through mechanical degrees of freedom of the scattering medium, as exploited in Raman and Brillouin scattering.

In this Letter, we create an arrangement allowing interaction between waves of arbitrary frequencies, which is achieved by introducing an additional coupling mechanism to impose artificial interaction between otherwise independent elements. Our "meta-atom" is based on a split-ring resonator (SRR), ${ }^{3}$ which became the prominent workhorse ${ }^{4}$ for microwave metamaterials, ${ }^{5-7}$ available in numerous varieties and implementations. ${ }^{8}$ To achieve a compact resonance and to avoid bianisotropy ${ }^{9}$ that complicates the response, ${ }^{10}$ we utilise the broadside-coupled SRR design. ${ }^{11}$

Being brought together, SRRs demonstrate a significant mutual coupling, ${ }^{12,13}$ resulting in a rich spectrum of magnetoinductive waves, ${ }^{13,14}$ specific bulk properties, ${ }^{12,15}$ and strong boundary effects in finite samples. ${ }^{16,17}$ While this coupling proves to be quite useful for application in magnetoinductive devices, ${ }^{18}$ imaging techniques, ${ }^{19-21}$ tunable (reconfigurable) metamaterials, ${ }^{22}$ and for design of nonlinear schemes, ${ }^{23}$ it is also of interest to realise a system where direct linear interaction is suppressed, while the strength of indirect mutual coupling is a nonlinear function of power.

To achieve this goal, we provide an additional interaction mechanism between the SRRs with the use of optical feedback (Fig. 1). Indeed, efficient optical tunability of the SRR response has already been reported in the terahertz ${ }^{24-28}$

\footnotetext{
${ }^{\text {a) }}$ Author to whom correspondence should be addressed. Electronic mail: mlapine@physics.usyd.edu.au
}

and microwave $\mathrm{e}^{29,30}$ domains; in particular, photodiodes (PDs) have been implemented to control the focusing properties of SRR arrays and to impose non-trivial competing nonlinearities. ${ }^{31}$

We now take an important step forward and provide a scheme where SRRs are actively involved in the optical control, serving as both receivers and sources of optical radiation. Our design comprises two varactor-loaded SRRs, which are arranged to be orthogonal to each other (Fig. 1). With this geometry, the symmetry of the arrangement ensures there is no electromagnetic coupling between the two resonators. However, one of the SRRs is controlled by a PD, and the other is equipped with a light-emitting diode (LED), positioned so as to provide efficient optical interaction between the LED and the PD. At low power of the electromagnetic signal launched into the LED-SRR, there is insufficient voltage rectified by the varactor to light up the LED, so there is no interaction between the SRRs. At higher power, the LED is lit and the emitted light is absorbed by the PD, providing a voltage across the varactor, thus changing its capacitance, which directly affects the resonance frequency of the PD-SRR. The higher the power, the brighter the LED, and the larger the voltage provided by the PD, causing a stronger change in the varactor capacitance. Thus, the resonant frequency of the PD-SRR is directly controlled by the

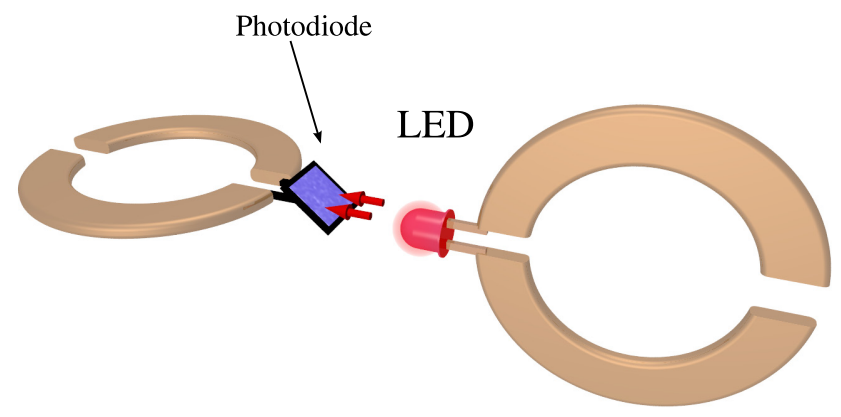

FIG. 1. Conceptual representation of photo-coupling in metamaterials: One resonator is capable of emitting light when excited with a microwave signal, while the other one has light-sensitive circuitry which affects its resonance. 
signal power in the LED-SRR, despite having no direct electromagnetic coupling between the two.

Furthermore, the designed SRR pair has a strong polarisation sensitivity. Indeed, when the pump wave is polarised so that the magnetic field is along the LED-SRR axis, the maximal current is excited and the LED is brightly lit, imposing the resonance frequency shift in the other SRR, as described above. However, for an orthogonal polarisation the LED-SRR is not excited at all, and no resonance shift is induced regardless of the pump power.

To demonstrate the efficiency of such coupling, we have fabricated an experimental prototype realised with this scheme (Fig. 2). Each "meta-atom" is a broadside-coupled SRR with copper strips $(1 \mathrm{~mm}$ width) printed on the two sides of an FR4 substrate (1 mm thickness). The two rings which constitute each SRR are identical; however, one of the SRRs is smaller then the other, so that the smaller one fits inside the larger one (Fig. 2(a)). The smaller SRR has an inner radius of $5 \mathrm{~mm}$ and the larger one of $6.05 \mathrm{~mm}$. Each ring of the SRR has a $1 \mathrm{~mm}$ gap, and an additional $0.5 \mathrm{~mm}$ gap where SMV1405 varactors (Skyworks) are mounted. In the smaller SRR, the varactors are biased by a pair of BPW34-S photodiodes (Opto Semiconductors). In the larger SRR, the varactors provide rectification, feeding the BL-L314URC LED (Betlux Electronics). Shunting between the varactors and PDs/LED was provided using $22 \mathrm{nH}$ chip inductors (LQG18HN22NJ00D, Murata), to decouple the photocomponents from the high-frequency microwave signals.

For the measurement of the resulting optically coupled SRR pair, we have designed two symmetric microstrip loop

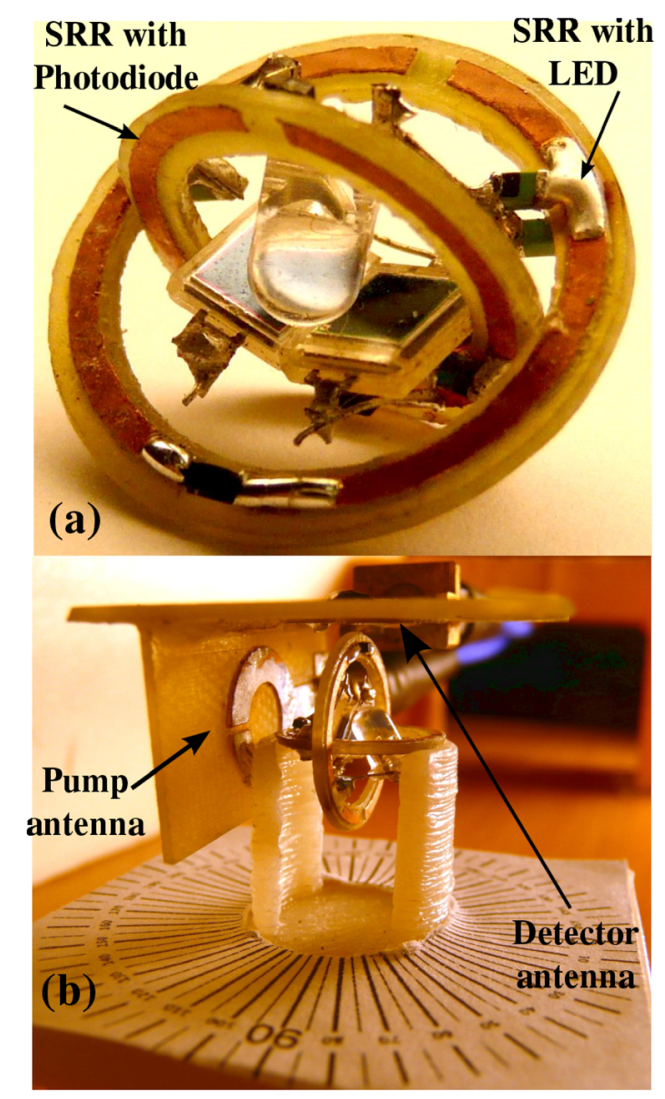

FIG. 2. Fabricated prototype of a "meta-atom" assembled as an optically coupled SRR pair; and a photograph of the experimental setup for studying power dependence and varying incident polarisation. antennas. The antennas were positioned in perpendicular planes and located in such a way that each antenna is only coupled to one of the rings in our element (see Fig. 2(b)), because the magnetic coupling to the other is cancelled due to symmetry reasons. In the configuration shown in Fig. 2(b), the LED-SRR is excited by the side antenna, which is connected to a signal generator (HP E8257D) through a signal amplifier (HP 83020A). The PD-SRR is probed by the upper antenna, connected to an Agilent PNA E8362C vector network analyser (VNA) to measure the reflection coefficient. All the experiments on the optical coupling were performed in the dark to prevent parasitic illumination of the PDs.

To check the power dependence, we rotated the SRR pair to achieve maximum excitation of the LED-SRR (at $90^{\circ}$ ), and measured the reflection spectra from the PD-SRR as a function of the power launched onto LED-SRR. The results, presented in Fig. 3(a), demonstrate a significant frequency shift of $18 \mathrm{MHz}$ when the power is increased to $33 \mathrm{dBm}$. Note that the frequency shift is not observed until a threshold at approximately $22 \mathrm{dBm}$, as lower power does not generate sufficient voltage to light the LED. The results are confirmed with complete circuit calculations of our design: In Fig. 3(b), we present the experimental dependence of the frequency shift as a function of power, compared with the circuit calculations.
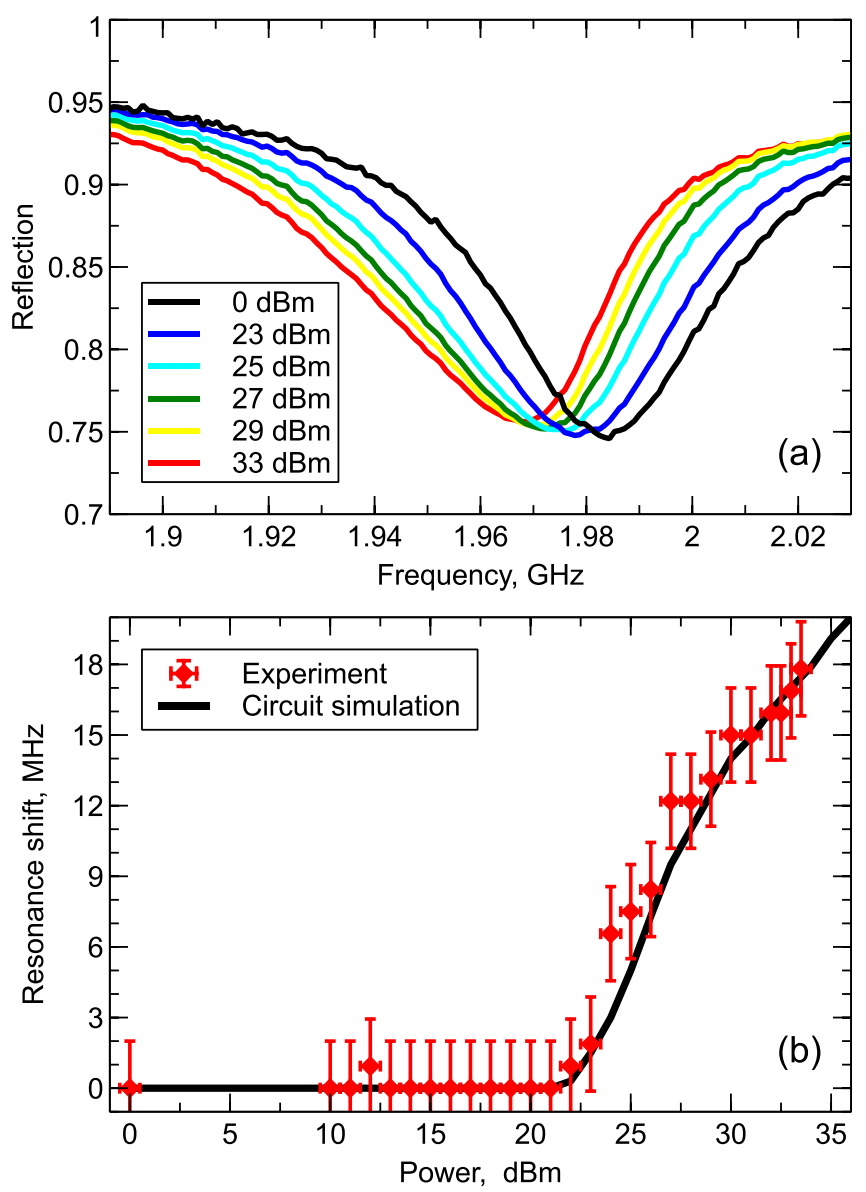

FIG. 3. Power dependence of the optically coupled SRR response. (a) Reflection spectra measured by the probe antenna at various power levels (see the inset) supplied to the pump antenna; (b) dependence of the frequency shift of the PD-SRR depending on the power launched into LEDSRR, experiment (squares) and simulation (solid curve). 

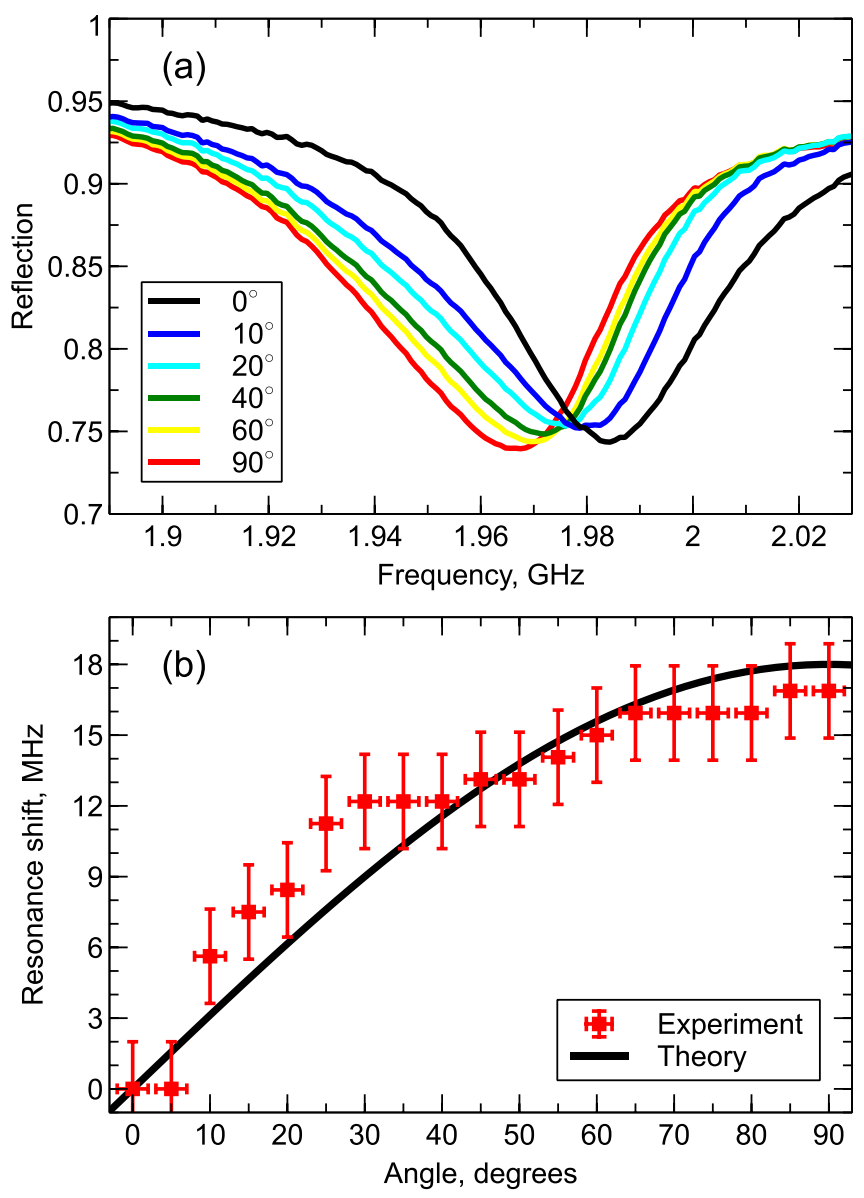

FIG. 4. Polarisation dependence of the optically coupled SRR response. (a) Reflection spectra measured by the probe antenna at various angles (see the inset) between the pump antenna and the LED-SRR; (b) dependence of the frequency shift of the PD-SRR on the orientation of the LED-SRR, experiment (squares) and theory (solid curve).

For the circuit simulations, we have used the nonlinear equivalent circuit of an SRR, reported earlier. ${ }^{31}$ The SRR itself has been represented by the self-inductance, capacitance, and resistance, and further loaded by a varactor. To represent the SMV1405 varactor, the corresponding SPICE model has been used. To find the appropriate linear parameters of the SRRs, we performed linear circuit simulations at low power, with the initial guess value for the SRR capacitance estimated analytically. ${ }^{5}$ Next, we performed nonlinear circuit simulations for the power from $0 \mathrm{dBm}$ to $35 \mathrm{dBm}$ in $1 \mathrm{~dB}$ steps. As a result, we obtained the voltage rectified by the varactors in the LED-SRR as a function of frequency for different power levels. When the power reaches $22 \mathrm{dBm}$, the voltage rectified by the varactors exceeds $1.5 \mathrm{~V}$, sufficient to light up the LED. At the power of $35 \mathrm{dBm}$ the voltage is over $2.2 \mathrm{~V}$, which makes the LED quite bright. The light from the LED shines onto the PD, which in turn imposes a voltage across the varactors in the PD-SRR, shifting its resonance frequency. The estimated frequency shift is of the order of $20 \mathrm{MHz}$.

To confirm the polarisation sensitivity, we performed an experiment with a fixed pump power of $32.5 \mathrm{dBm}$ while gradually rotating the SRR pair between the positions with the LED-SRR orthogonal to the pump antenna (at $\phi=0^{\circ}$ ) and parallel to it (at $\phi=90^{\circ}$ ). As expected, the LED was not lit at $0^{\circ}$ and no resonance shift was detected, while the gradual change of polarisation angle towards $90^{\circ}$ resulted in the displaced spectra (Fig. 4(a)) with the resonance shift varying with angle in accordance with the expected proportionality to $\cos \phi$, as shown in Fig. 4(b).

The designed meta-atom opens non-standard opportunities to impose an optical control mechanism over microwave signals. For example, a chain of such elements supports two initially independent magnetoinductive waves with orthogonal polarisations, which can propagate along the chain without direct interaction: one of the waves only drives the sequence of PD-SRRs while the other one only drives the sequence of the LED-SRRs. However, by varying power of wave transmitted with the LED-SRR polarisation, we will launch an optical feedback onto the chain of PD-SRRs and thus alter its dispersion characteristics despite the lack of coupling between the two subsystems in the linear regime. This feedback mechanism can be attractive for applications in signal processing and filtering, as well as for tuning and sensing.

In summary, we have designed and experimentally implemented a meta-atom with nonlinear interaction between microwave signals via an optical channel. Our approach allows interaction to be imposed between otherwise independent signals with arbitrary frequencies, and therefore significantly widens the range of nonlinear processes possible in such optically-coupled systems.

The authors are grateful to Klaus Betzler and Maxim Gorkunov for preliminary discussions, to Stanislav Maslovski for helpful advice, and to Igor Y. Denisiuk for experimental equipment. This work was supported by the Ministry of Education and Science of Russian Federation (Project No. 11.G34.31.0020), Russian Foundation for Basic Research (Projects No. 13-02-00411 and 14-02-31783), Scholarship of the President of the Russian Federation for young scientists and graduate students, and by the Australian Research Council (CUDOS Centre of Excellence CE110001018). A.P.S. acknowledges the support of SPIE Scholarship 2012-2013 and the IEEE MTT-S Undergraduate Scholarship for spring 2013. A.P.S. and D.S.F. are also grateful to the "Dynasty" Foundation (Russia).

${ }^{1}$ N. Bloembergen, Nonlinear Optics (Benjamin, New York, 1965).

${ }^{2} \mathrm{~J}$. Helszajn, Passive and Active Microwave Circuits (Wiley, New York, 1978).

${ }^{3}$ W. N. Hardy and L. A. Whitehead, Rev. Sci. Instrum. 52, 213 (1981).

${ }^{4}$ J. B. Pendry, A. J. Holden, D. J. Robbins, and W. J. Stewart, IEEE Trans. Microw. Theory Tech. 47, 2075 (1999).

${ }^{5}$ R. Marqués, F. Martín, and M. Sorolla, Metamaterials with Negative Parameters (Wiley, 2008).

${ }^{6}$ L. Solymar and E. Shamonina, Waves in Metamaterials (Oxford University Press, 2009).

${ }^{7}$ Handbook on Metamaterials, edited by F. Capolino (CRC Press, 2009).

${ }^{8}$ R. Marqués, L. Jelinek, M. Freire, J. Baena, and M. Lapine, Proc. IEEE 99, 1660 (2011).

${ }^{9}$ A. Serdyukov, I. Semchenko, S. Tretyakov, and A. Sihvola, Electromagnetics of Bi-anisotropic Materials: Theory and Applications (Gordon and Breach, Amsterdam, 2001).

${ }^{10}$ B. Sauviac, C. R. Simovski, and S. A. Tretyakov, Electromagnetics 24, 317 (2004).

${ }^{11}$ R. Marqués, F. Mesa, J. Martel, and F. Medina, IEEE Trans. Antennas Propag. 51, 2572 (2003).

${ }^{12}$ M. Gorkunov, M. Lapine, E. Shamonina, and K. H. Ringhofer, Eur. Phys. J. B 28, 263 (2002). 
${ }^{13}$ E. Shamonina, V. A. Kalinin, K. H. Ringhofer, and L. Solymar, J. Appl. Phys. 92, 6252 (2002).

${ }^{14}$ R. R. A. Syms, E. Shamonina, V. Kalinin, and L. Solymar, J. Appl. Phys. 97, 064909 (2005).

${ }^{15}$ J. D. Baena, L. Jelinek, R. Marqués, and M. Silveirinha, Phys. Rev. A 78, 013842 (2008).

${ }^{16}$ M. Lapine, L. Jelinek, M. Freire, and R. Marqués, Phys. Rev. B 82, 165124 (2010).

${ }^{17}$ M. Lapine, L. Jelinek, and R. Marqués, Opt. Express 20, 18297 (2012).

${ }^{18}$ R. R. A. Syms, E. Shamonina, and L. Solymar, IEEE Proc. Microwaves, Antennas Propag. 153, 111 (2006).

${ }^{19}$ R. Syms, T. Floume, I. Young, L. Solymar, and M. Rea, Metamaterials 4, 1 (2010).

${ }^{20}$ M. Freire, L. Jelinek, R. Marqués, and M. Lapine, J. Magn. Reson. 203, 81 (2010).

${ }^{21}$ R. Syms, E. Shamonina, and L. Solymar, Metamaterials 5, 8 (2011).

${ }^{22}$ M. Lapine, D. Powell, M. Gorkunov, I. Shadrivov, R. Marqués, and Y. Kivshar, Appl. Phys. Lett. 95, 084105 (2009).
${ }^{23}$ M. Lapine, I. V. Shadrivov, D. A. Powell, and Y. S. Kivshar, Nature Mater. 11, 30 (2012).

${ }^{24}$ W. J. Padilla, A. J. Taylor, C. Highstrete, M. Lee, and R. D. Averitt, Phys. Rev. Lett. 96, 107401 (2006).

${ }^{25}$ R. D. Chowdhury, R. Singh, J. O'Hara, H.-T. Chen, A. Taylor, and A. Azad, Appl. Phys. Lett. 99, 231101 (2011).

${ }^{26}$ J. Gu, R. Singh, X. Liu, X. Zhang, Y. Ma, S. Zhang, S. Maier, Z. Tian, A. Azad, H.-T. Chen et al., Nat. Commun. 3, 1151 (2012).

${ }^{27}$ M. Kafesaki, N. H. Shen, S. Tzortzakis, and C. M. Soukoulis, J. Opt. 14, 114008 (2012).

${ }^{28}$ D. Shrekenhamer, C. M. Watts, and W. J. Padilla, Opt. Express 21, 12507 (2013).

${ }^{29}$ P. Kapitanova, S. Maslovski, I. Shadrivov, P. Voroshilov, D. Filonov, P. Belov, and Y. Kivshar, Appl. Phys. Lett. 99, 251914 (2011).

${ }^{30}$ I. V. Shadrivov, P. V. Kapitanova, S. I. Maslovski, and Y. S. Kivshar, Phys. Rev. Lett. 109, 083902 (2012).

${ }^{31}$ P. V. Kapitanova, A. P. Slobozhanyuk, I. V. Shadrivov, P. A. Belov, and Y. S. Kivshar, Appl. Phys. Lett. 101, 231904 (2012). 